\title{
Robust Frequency Hopping for Interference and Fading Channels
}

\author{
Don Torrieri, Shi Cheng, and Matthew C. Valenti
}

\begin{abstract}
A robust frequency-hopping system with noncoherent detection, iterative turbo decoding and demodulation, and channel estimation is presented. The data modulation is the spectrally compact nonorthogonal continuous-phase frequency-shift keying, which strengthens the frequency-hopping system against multiple-access interference and multitone jamming. An analysis based on information theory provides the optimal values of the modulation index when there is a bandwidth constraint. The channel estimator, which is derived by applying the expectationmaximization algorithm, accommodates both frequency-selective fading and interference. Simulation experiments demonstrate the excellent system performance against both partial-band and multiple-access interference.
\end{abstract}

Index Terms-Frequency hopping, partial-band interference, multiple-access interference, frequency-selective fading, continuous-phase frequency-shift keying.

\section{INTRODUCTION}

$\mathbf{T}$ HIS paper describes and analyzes a robust frequencyhopping system with noncoherent detection, iterative turbo decoding and demodulation, and channel estimation. The system is designed to be effective not only when operating over the additive white Gaussian noise (AWGN) and fading channels but also in environments with multiple-access interference and multitone jamming.

Noncoherent or differentially coherent demodulation has practical advantages and is often necessary because of the difficulty of phase estimation after every frequency hop. A common choice of modulation is orthogonal frequency-shift keying (FSK). With orthogonal FSK, the energy efficiency can be improved by increasing the alphabet size $q$ [1], which is equal to the number of possible transmit frequencies in the signal set during each hop dwell interval. The problem is that a large bandwidth $B_{u}$ of each frequency channel, although necessary to support a large number of transmit frequencies, reduces the number of frequency channels available when the hopping is over a spectral region with fixed bandwidth $W$. This reduction makes the system more vulnerable to both multiple-access frequency-hopping signals and multitone jamming [2]. A reduction in $B_{u}$ is obtained by using nonorthogonal continuous-phase frequency-shift keying (CPFSK).

Paper approved by G. E. Corazza, the Editor for Spread Spectrum of the IEEE Communications Society. Manuscript received September 27, 2006; revised April 3, 2007 and September 27, 2007. This paper was presented in part at the IEEE International Conference on Communications, Glasgow, Scotland, June 2007.

D. Torrieri is with the US Army Research Laboratory, Adelphi, MD (email: dtorr@arl.army.mil).

S. Cheng is with ArrayComm LLC, San Jose, CA (e-mail: shi.cheng@gmail.com).

M. C. Valenti is with West Virginia University, Morgantown, WV (e-mail: mvalenti@csee.wvu.edu).

Digital Object Identifier 10.1109/TCOMM.2008.060549.
As an example of the importance of $B_{u}$, consider multitone jamming of a frequency-hopping system with $q$-ary CPFSK in which the thermal noise is absent and each jamming tone has its carrier frequency within a distinct frequency channel. The uncoded symbol-error probability is approximately [2]

$$
P_{s}=\left(\frac{q-1}{q}\right) B_{u} T_{b}\left(\frac{\mathcal{E}_{b}}{I_{t 0}}\right)^{-1}, \quad B_{u} T_{b} \leq \frac{\mathcal{E}_{b}}{I_{t 0}} \leq W T_{b}
$$

where $\mathcal{E}_{b}$ is the energy per bit, $T_{b}$ is the bit duration, $I_{t 0} W$ is the total jamming power, and $B_{u}$ is the uncoded bandwidth. This equation indicates the significant benefit of a small bandwidth in reducing the effect of multitone jamming.

Robust system performance is provided by using nonorthogonal CPFSK, a turbo code, bit-interleaved coded modulation (BICM) [3], iterative decoding and demodulation, and channel estimation. The bandwidth of $q$-ary CPFSK decreases with reductions in the modulation index $h$. Although the lack of orthogonality when $h<1$ will cause a performance loss for the AWGN and fading channels, the turbo decoder makes this loss minor compared with the gain against multiple-access interference and multitone jamming.

Frequency hopping with binary orthogonal FSK, a turbo product code, and perfect channel information has been examined in [4]. Frequency hopping with differential $q$-ary phase-shift keying, iterative decoding, and channel estimation has been analyzed in [5], [6]. The proposed system with noncoherent, nonorthogonal CPFSK has the following primary advantages relative to other proposed systems with differential detection, coherent detection, or orthogonal modulation.

1. No extra reference symbol and no estimation of the phase offset in each dwell interval are required.

2. It is not necessary to assume that the phase offset is constant throughout a dwell interval.

3. The channel estimators are much more accurate and can estimate an arbitrary number of interference and noise spectral-density levels.

4. The compact spectrum during each dwell interval allows more frequency channels and, hence, enhances performance against multiple-access interference and multitone jamming.

5. Because noncoherent detection is used, system complexity is independent of the choice of $h$, and thus there is much more design flexibility than is possible in coherent CPFSK systems.

Section II presents the basic system model. The demodulator for noncoherent, nonorthogonal CPFSK is described and demodulator bit metrics are derived in Section III. The channel estimator is derived in Section IV by applying the expectation-maximization algorithm. In Section V, an analysis 


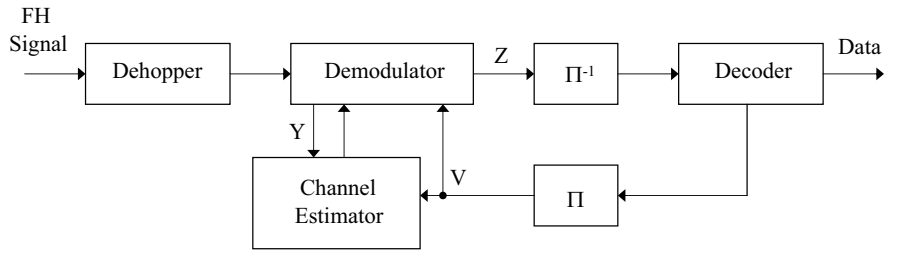

Fig. 1. Architecture of receiver for frequency-hopping system with turbo code. $\Pi=$ interleaver; $\Pi^{-1}=$ deinterleaver.

based on information theory provides the optimal values of the modulation index when there is a bandwidth constraint. The simulation experiments of Sections VI and VII demonstrate the excellent system performance against both partial-band and multiple-access interference.

\section{SyStem MOdel}

In the transmitter of the proposed system, which uses BICM, encoded message bits are interleaved and then placed into a length- $N_{d}$ vector $\mathbf{d}$ with elements $d_{i} \in\{1,2, \ldots, q\}$, each of which represents $m=\log _{2} q$ bits. The vector $\mathbf{d}$ generates the sequence of tones that are frequency-translated by the carrier frequency of the frequency-hopping waveform during a signaling interval. After the modulated signal passes through an AWGN or fading channel with partial-band or multiple-access interference, the receiver front-end dehops the signal, as shown in Fig. 1. The dehopped signal passes through a bank of $q$ matched filters, each of which is implemented as a quadrature pair [1], [2]. The output of each matched filter is sampled at the symbol rate to produce a sequence of complex numbers. Assuming that symbol synchronization exists, the complex samples are then placed into an $q \times N_{d}$ matrix $\mathbf{Y}$ whose $i^{t h}$ column represents the outputs of the matched filters corresponding to the $i^{t h}$ received symbol. The matrix $\mathbf{Y}$ is applied to the channel estimator and is used to produce an $m \times N_{d}$ matrix $\mathbf{Z}$ of demodulator bit metrics.

The demodulator exchanges information with both the turbo decoder and the channel estimators. After deinterleaving, the demodulator bit metrics are applied to the decoder. The decoder feeds a priori information (in the form of an $m \times N_{d}$ matrix $\mathbf{V}$ of decoder bit metrics) back to the demodulator and channel estimator, in accordance with the turbo principle. Frequency-selective fading changes the fading amplitude from hop to hop, and the partial-band and multiple-access interference change the interference and noise during some hop dwell intervals. Consequently, estimates of the fading amplitude and the spectral density of the interference and noise are computed for a block size $\mathrm{N}$ that is smaller than or equal to the number of symbols in the hop dwell interval. If there are $N$ symbols per block, then there are $\left\lceil N_{d} / N\right\rceil$ blocks per codeword.

\section{Demodulator Metrics}

The complex envelope of a unit-energy $q$-ary CPFSK symbol waveform with zero initial phase offset is

$$
s_{l}(t)=\frac{1}{\sqrt{T_{s}}} e^{j 2 \pi l h t / T_{s}}, \quad 0 \leq t \leq T_{s}, \quad l=1,2, \ldots, q
$$

where $T_{s}$ is the symbol duration and $h$ is the modulation index. Because of the continous-phase constraint, the initial phase of CPFSK symbol $i$ is $\phi_{i}=\phi_{i-1}+2 \pi l h$. The phase continuity ensures the compact spectrum of the CPFSK waveform. Suppose that symbol $i$ of a codeword uses unitenergy waveform $s_{d_{i}}(t)$, where the integer $d_{i}$ is a function of the codeword. If this codeword is transmitted over a channel with fading and additive Gaussian noise, the received signal for symbol $i$ can be expressed in complex notation as

$$
\begin{aligned}
r_{i}(t) & =\operatorname{Re}\left[a_{i} \sqrt{2 \mathcal{E}_{s}} s_{d_{i}}(t) e^{j 2 \pi f_{c} t+\theta_{i}}\right]+n_{i}(t), \quad 0 \leq t \leq T_{s} \\
i & =1,2, \ldots, N_{d}
\end{aligned}
$$

where $n_{i}(t)$ is independent, zero-mean, white Gaussian noise with two-sided power spectral density $N_{0 i} / 2, f_{c}$ is the carrier frequency, $\theta_{i}$ is the phase, $\mathcal{E}_{s}$ is the signal energy, and $a_{i}$ accounts for the fading amplitude. Without loss of generality, we assume $E\left[a_{i}^{2}\right]=1$ so that the average received symbol energy is $\mathcal{E}_{s}$. The phase $\theta_{i}$ is the phase due to the contributions of the CPFSK constraint, the fading, and the frequency offset of the receiver. One might consider exploiting the inherent memory in the CPFSK when computing the metric transferred from the demodulator to a decoder, as described in [7]. However, phase stability over several symbols is necessary, and the demodulator functions as a rate-one inner decoder. Furthermore, a trellis demodulator requires a rational $h$ and the number of states depends on the denominator of $h$. More design flexibility exists if the demodulator metrics are computed on a symbol-by-symbol basis, and the memory in the turbo code is exploited rather than the memory in the modulation.

Matched-filter $k$, which is matched to $s_{k}(t)$, produces the output samples

$$
\begin{aligned}
y_{k, i} & =\sqrt{2} \int_{0}^{T_{s}} r_{i}(t) e^{-j 2 \pi f_{c} t} s_{k}^{*}(t) d t, \\
i & =1,2, \ldots, N_{d}, \quad k=1,2, \ldots, q .
\end{aligned}
$$

The substitution of (3) into (4) and the approximation that each of the $\left\{s_{k}(t)\right\}$ has a spectrum confined to $|f|<f_{c}$ yields

$$
y_{k, i}=a_{i} \sqrt{\mathcal{E}_{s}} e^{j \theta_{i}} \rho_{d_{i}-k}+n_{k, i}
$$

where

$$
n_{k, i}=\sqrt{2} \int_{0}^{T_{s}} n_{i}(t) e^{-j 2 \pi f_{c} t} s_{k}^{*}(t) d t
$$

and

$$
\rho_{l}=\frac{\sin (\pi h l)}{\pi h l} e^{j \pi h l} .
$$

Since $n_{i}(t)$ is zero-mean and white and the spectra of the $\left\{s_{k}(t)\right\}$ are confined, it follows that each $n_{k, i}$ is zero-mean,

$$
E\left[n_{k, i} n_{l, i}^{*}\right]=N_{0 i} \rho_{l-k}
$$

and the $\left\{n_{k, i}\right\}$ have circular symmetry:

$$
E\left[n_{k, i} n_{l, i}\right]=0 .
$$

Since $n_{i}(t)$ is a Gaussian process, the real and imaginary components of $n_{k, i}$ are jointly Gaussian, and the set $\left\{n_{k, i}\right\}$ comprises complex-valued jointly Gaussian random variables.

Let $\mathbf{y}_{i}=\left[y_{1, i} \ldots y_{q, i}\right]^{T}$ denote the column vector of the matched-filter outputs corresponding to symbol $i$, and let $\mathbf{n}=\left[n_{1, i} \ldots n_{q, i}\right]^{T}$. Then given that the transmitted symbol 
is $d_{i}$, the symbol energy is $\mathcal{E}_{s}$, the fading amplitude is $a_{i}$, the noise spectral density is $N_{0 i}$, and the phase is $\theta_{i}, \mathbf{y}_{i}=\overline{\mathbf{y}}_{i}+\mathbf{n}$, where $\overline{\mathbf{y}}_{i}=E\left[\mathbf{y}_{i} \mid d_{i}, \mathcal{E}_{s}, a_{i}, N_{0 i}, \theta_{i}\right]$. Equation (5) indicates that the kth component of $\overline{\mathbf{y}}_{i}$ is

$$
\bar{y}_{k, i}=a_{i} \sqrt{\mathcal{E}_{s}} e^{j \theta_{i}} \rho_{d_{i}-k} .
$$

The covariance matrix of $\mathbf{y}_{i}$ is

$$
\begin{aligned}
\mathbf{R}_{i} & =E\left[\left(\mathbf{y}_{i}-\overline{\mathbf{y}}_{i}\right)\left(\mathbf{y}_{i}-\overline{\mathbf{y}}_{i}\right)^{H} \mid d_{i}, a_{i} \sqrt{\mathcal{E}_{s}}, N_{0 i}, \theta_{i}\right] \\
& =E\left[\mathbf{n n}^{H}\right]
\end{aligned}
$$

and its elements are given by (8). It is convenient to define the matrix $\mathbf{K}=\mathbf{R} / N_{0 i}$ with components

$$
K_{k l}=\rho_{l-k} .
$$

We can represent the conditional probability density function of $\mathbf{y}_{i}$ given that the transmitted symbol is $d_{i}$, the symbol energy is $\mathcal{E}_{s}$, the fading amplitude is $a_{i}$, the noise spectral density is $N_{0 i}$, and the phase is $\theta_{i}$ as

$$
\begin{aligned}
& p\left(\mathbf{y}_{i} \mid d_{i}, a_{i} \sqrt{\mathcal{E}_{s}}, N_{0 i}, \theta_{i}\right) \\
& =\frac{1}{\pi^{q} N_{0 i}^{q} \operatorname{det} \mathbf{K}} \exp \left[-\frac{1}{N_{0 i}}\left(\mathbf{y}_{i}-\overline{\mathbf{y}}_{i}\right)^{H} \mathbf{K}^{-1}\left(\mathbf{y}_{i}-\overline{\mathbf{y}}_{i}\right)\right]
\end{aligned}
$$

where $\mathbf{K}$ is independent of $\left(d_{i}, \mathcal{E}_{s}, a_{i}, N_{0 i}, \theta_{i}\right)$.

An expansion of the quadratic in (13) yields

$$
\begin{aligned}
Q_{i} & =\left(\mathbf{y}_{i}-\overline{\mathbf{y}}_{i}\right)^{H} \mathbf{K}^{-1}\left(\mathbf{y}_{i}-\overline{\mathbf{y}}_{i}\right) \\
& =\mathbf{y}_{i}{ }^{H} \mathbf{K}^{-1} \mathbf{y}_{i}+\overline{\mathbf{y}}_{i}{ }^{H} \mathbf{K}^{-1} \overline{\mathbf{y}}_{i}-2 \operatorname{Re}\left(\mathbf{y}_{i}{ }^{H} \mathbf{K}^{-1} \overline{\mathbf{y}}_{i}\right) .
\end{aligned}
$$

Equations (10) and (12) indicate that $\overline{\mathbf{y}}_{i}$ is proportional to the $d_{i}$ th column of $\mathbf{K}$ :

$$
\overline{\mathbf{y}}_{i}=a_{i} \sqrt{\mathcal{E}_{s}} e^{j \theta_{i}} \mathbf{K}_{:, d_{i}} .
$$

Since $\mathbf{K}^{-1} \mathbf{K}=\mathbf{I}$, only the $d_{i}$ th component of the column vector $\mathbf{K}^{-1} \overline{\mathbf{y}}_{i}$ is nonzero and

$$
Q_{i}=\mathbf{y}_{i}{ }^{H} \mathbf{K}^{-1} \mathbf{y}_{i}+a_{i}^{2} \mathcal{E}_{s}-2 a_{i} \sqrt{\mathcal{E}_{s}} \operatorname{Re}\left(y_{d_{i}, i} e^{-j \theta_{i}}\right) .
$$

For noncoherent signals, it is assumed that each $\theta_{i}$ is uniformly distributed over $[0,2 \pi)$. Substituting (16) into (13), expressing $y_{d_{i}, i}$ in polar form, and integrating over $\theta_{i}$, we obtain the probability density function

$$
\begin{aligned}
p\left(\mathbf{y}_{i} \mid d_{i}, a_{i} \sqrt{\mathcal{E}_{s}}, N_{0 i}\right) & =\frac{\exp \left(-\frac{\mathbf{y}_{i}{ }^{H} \mathbf{K}^{-1} \mathbf{y}_{i}+a_{i}^{2} \mathcal{E}_{s}}{N_{0 i}}\right)}{\pi^{q} N_{0 i}^{q} \operatorname{det} \mathbf{K}} \\
& \times I_{0}\left(\frac{2 a_{i} \sqrt{\mathcal{E}_{s}}\left|y_{d_{i}, i}\right|}{N_{0 i}}\right)
\end{aligned}
$$

where $I_{0}()$ is the modified Bessel function of the first kind and order zero. Since the white noise $n_{i}(t)$ is independent from symbol to symbol, $\mathbf{y}_{i}$ with the density given by (17) is independent of $\mathbf{y}_{l}, i \neq l$.

Let $\widehat{A}$ and $\widehat{B}$ denote the estimates of $A=N_{0}$ and $B=$ $2 a \sqrt{\mathcal{E}_{s}}$, respectively, for a dwell interval of $N$ symbols during which $a_{i}=a$ and $N_{0 i}=N_{0}$ are constants. Let $b_{k, i}$ denote bit $k$ of symbol $i$. Let $\mathbf{Z}$ denote the $m \times N_{d}$ matrix whose element $z_{k, i}$ is the log-likelihood ratio for $b_{k, i}$ computed by the demodulator. The matrix $\mathbf{Z}$ is reshaped into a row vector and deinterleaved, and the resulting vector $\mathbf{z}^{\prime}$ is fed into the turbo decoder. The extrinsic information $\mathbf{v}^{\prime}$ at the output of the decoder is interleaved and reshaped into a $m \times N_{d}$ matrix $\mathbf{V}$ containing the a priori information:

$$
v_{k, i}=\log \frac{p\left(b_{k, i}=1 \mid \mathbf{Z} \backslash z_{k, i}\right)}{p\left(b_{k, i}=0 \mid \mathbf{Z} \backslash z_{k, i}\right)}
$$

where conditioning on $\mathbf{Z} \backslash z_{k, i}$ means that the extrinsic information for bit $b_{k, i}$ is produced without using $z_{k, i}$. Since $\mathbf{V}$ is fed back to the demodulator,

$$
z_{k, i}=\log \frac{p\left(b_{k, i}=1 \mid \mathbf{y}_{i}, \gamma_{\lceil i / N\rceil}^{\prime}, \mathbf{v}_{i} \backslash v_{k, i}\right)}{p\left(b_{k, i}=0 \mid \mathbf{y}_{i}, \gamma_{\lceil i / N\rceil}^{\prime}, \mathbf{v}_{i} \backslash v_{k, i}\right)}
$$

where $\gamma^{\prime}=\{\hat{A}, \hat{B}\}$. Partition the set of symbols $\mathcal{D}=$ $\{1, \ldots, q\}$ into two disjoint sets $\mathcal{D}_{k}^{(1)}$ and $\mathcal{D}_{k}^{(0)}$, where $\mathcal{D}_{k}^{(b)}$ contains all symbols labelled with $b_{k}=b$. The extrinsic information can then be expressed as [8], [9]

$$
z_{k, i}=\log \frac{\sum_{d \in \mathcal{D}_{k}^{(1)}} p\left(\mathbf{y}_{i} \mid d, \gamma_{\lceil i / N\rceil}^{\prime}\right) \prod_{\substack{j=1 \\ j \neq k}}^{m} \exp \left(b_{j}(d) v_{j, i}\right)}{\sum_{d \in \mathcal{D}_{k}^{(0)}} p\left(\mathbf{y}_{i} \mid d, \gamma_{\lceil i / N\rceil}^{\prime}\right) \prod_{\substack{j=1 \\ j \neq k}}^{m} \exp \left(b_{j}(d) v_{j, i}\right)}
$$

where $b_{j}(d)$ is the value of the $j^{\text {th }}$ bit in the labelling of symbol $d$. Substituting (17) into (20) and cancelling common factors, we obtain

$$
z_{k, i}=\log \frac{\sum_{d \in \mathcal{D}_{k}^{(1)}} I_{0}\left(\gamma_{\lceil i / N\rceil}\left|y_{d_{i}, i}\right|\right) \prod_{\substack{j=1 \\ j \neq k}}^{m} \exp \left(b_{j}(d) v_{j, i}\right)}{\sum_{d \in \mathcal{D}_{k}^{(0)}} I_{0}\left(\gamma_{\lceil i / N\rceil}\left|y_{d_{i}, i}\right|\right) \prod_{\substack{j=1 \\ j \neq k}}^{m} \exp \left(b_{j}(d) v_{j, i}\right)}
$$

where only the ratio $\gamma=\hat{B} / \hat{A}$ is needed rather than the individual estimates.

\section{Channel Estimators}

Since under block fading and time-varying interference, $A$ and $B$ can change on a block-by-block basis, each block is processed separately and in an identical fashion. To maintain robustness, the estimators make no assumptions regarding the distribution of the quantities to be estimated, nor do they make any assumptions regarding the correlation from block to block. The estimators directly use the channel observation for a single block while the observations of the other blocks are used indirectly through feedback of extrinsic information from the decoder. Thus in this section, $\mathbf{Y}$ is a generic $q \times N$ received block, $\mathbf{d}=\left[d_{1}, \ldots, d_{N}\right]$ is the corresponding set of transmitted symbols, and $\{\hat{A}, \hat{B}\}$ is the corresponding set of channel estimators.

Rather than attempting to directly evaluate the maximumlikelihood estimates, the expectation-maximization (EM) algorithm can be used as an iterative approach to estimation [10]. Let $\{\mathbf{Y}, \mathbf{d}\}$ denote the complete data set. Since $\log p(\mathbf{d})$ is independent of $A$ and $B$ and, hence, does not affect the maximization, the log-likelihood of the complete data set is $L(A, B)=\log p(\mathbf{Y}, \mathbf{d} \mid A, B)=\log p(\mathbf{Y} \mid \mathbf{d}, A, B)+$ $\log p(\mathbf{d}) \sim \log p(\mathbf{Y} \mid \mathbf{d}, A, B)$. 
Since $\mathbf{y}_{i}$ and $\mathbf{y}_{l}$ are independent for $i \neq l$,(17) implies that

$$
\begin{aligned}
& p(\mathbf{Y} \mid \mathbf{d}, A, B) \\
& =\frac{\exp \left[-\frac{D}{A}-\frac{N B^{2}}{4 A}+\sum_{i=1}^{N} \log I_{0}\left(\frac{B\left|y_{d_{i}, i}\right|}{A}\right)\right]}{\left(\pi^{q} A^{q} \operatorname{det} \mathbf{K}\right)^{N}}
\end{aligned}
$$

where

$$
D=\sum_{i=1}^{N} \mathbf{y}_{i}{ }^{H} \mathbf{K}^{-1} \mathbf{y}_{i} .
$$

After dropping irrelevant constants, we obtain

$$
L(A, B) \sim-q N \log A-\frac{D}{A}-\frac{N B^{2}}{4 A}+\sum_{i=1}^{N} \log I_{0}\left(\frac{B\left|y_{d_{i}, i}\right|}{A}\right) .
$$

The form of this equation indicates that the parameters $A$ and $B$ must both be estimated rather than just the ratio $B / A$.

Let $r$ denote the EM iteration number, and $\hat{A}^{(r)}, \hat{B}^{(r)}$ the estimates of $A, B$ during the $r^{\text {th }}$ iteration. Applying the expectation step (E-step) and the maximization step (M-step) with (24) in a similar manner to that in [11], it is found that

$$
\begin{aligned}
& \hat{A}^{(r)}=\frac{1}{q N}\left(D-\frac{N\left(\hat{B}^{(r)}\right)^{2}}{4}\right) \\
& \hat{B}^{(r)}=\frac{2}{N} \sum_{i=1}^{N} \sum_{k=1}^{q} p_{k, i}^{(r-1)}\left|y_{k, i}\right| F\left(\frac{4 q N \hat{B}^{(r)}\left|y_{k, i}\right|}{4 D-N\left(\hat{B}^{(r)}\right)^{2}}\right)
\end{aligned}
$$

where $F(x)=I_{1}(x) / I_{0}(x), I_{1}(x)$ is the modified Bessel function of the first kind and order one,

$$
p_{k, i}^{(r-1)}=\alpha_{i}^{(r-1)} I_{0}\left(\frac{\hat{B}^{(r-1)}\left|y_{k, i}\right|}{\hat{A}^{(r-1)}}\right) p\left(d_{i}=k\right)
$$

$\alpha_{i}^{(r-1)}$ is the normalization factor forcing $\sum_{k=1}^{q} p_{k, i}^{(r-1)}=1$, i.e.,

$$
\alpha_{i}^{(r-1)}=\frac{1}{\sum_{k=1}^{q} I_{0}\left(\frac{\hat{B}^{(r-1)}\left|y_{k, i}\right|}{\hat{A}^{(r-1)}}\right) p\left(d_{i}=k\right)}
$$

and $p\left(d_{i}=k\right)$ is the probability that $d_{i}=k$ estimated by the decoder.

While a closed form solution to (26) is difficult to obtain, it can be found recursively [12]. The recursion involves initially replacing $\hat{B}^{(r)}$ on the right-hand side of (26) with $\hat{B}^{(r-1)}$ from the previous EM iteration. To select an initial estimate for $B$, consider what happens in the absence of noise. Without noise, (5) implies that either $\left|y_{k, i}\right|=a \sqrt{\mathcal{E}_{s}}$ (when $k=d_{i}$ ) or $\left|y_{k, i}\right|=0$ (otherwise). Thus, an estimate for $a \sqrt{\mathcal{E}_{s}}=B / 2$ can be achieved by taking the maximum $\left|y_{k, i}\right|$ over any column of $\mathbf{Y}$. To account for the possibility of noise, the average can be taken across all columns in the block, resulting in

$$
\hat{B}^{(0)}=\frac{2}{N} \sum_{i=1}^{N} \max _{k}\left|y_{k, i}\right| \text {. }
$$

The initial estimate of $A$ is found from $\hat{B}^{(0)}$ by evaluating (25) for $r=0$. After the initial values $\hat{A}^{(0)}$ and $\hat{B}^{(0)}$ are calculated, the initial probabilities $\left\{p_{k, i}^{(0)}\right\}$ are calculated from (27) and
(28). The EM algorithm terminates when $\hat{B}^{(r)}$ converges to some fixed value, typically in fewer than 10 EM iterations.

The complexity of the estimator is as follows. The initial estimate of $\hat{B}$ calculated using (29) requires $N$ maximizations over $q$ values, $N-1$ additions, and a single multiplication by $2 / N$. The calculation of $D$ in (23), which only needs to be computed once prior to the first EM iteration, requires $N q(q+1)$ multiplications and $N q^{2}-1$ additions. For each EM iteration, the calculation $\hat{A}^{(r)}$ using (25) requires only two multiplications and an addition. Calculating $p_{k, i}^{(r-1)}$ using (27) and (28) requires $3 N q+1$ multiplications, $N(q-1)$ additions, and $N q$ lookups of the $I_{0}(\cdot)$ function. Calculation of $\hat{B}^{(r)}$ by solving (26) is recursive, and complexity depends on the number of recursions for each value of $r$. Suppose that there are $\xi$ recursions, then the calculation will require $N q+\xi(2 N q+4)$ multiplications, $\xi N q$ additions, and $\xi N q$ lookups of the $F(\cdot)$ function. A stopping criterion is used for the calculation of $\hat{B}$ such that the recursions stop once $\hat{B}$ is within $10 \%$ of its value during the previous recursion or a maximum number of 10 recursions is reached. With such a stopping criterion, an average of only about 2 or 3 recursions are required.

\section{Selection of Modulation Index}

Let $B_{\max }$ denote the maximum bandwidth of the CPFSK modulation such that the hopping band accommodates enough frequency channels to ensure adequate performance against multiple-access interference and multitone jamming. We seek to determine the values of $h, q$, and code-rate $R$ of the turbo code that provide a good performance over the fading and AWGN channels in the presence of partial-band interference. For specific values of the modulation parameters $h$ and $q$, the code rate is limited by the bandwidth requirement. Let $B_{u} T_{b}$ denote the normalized, 99-percent power bandwidth of the uncoded CPFSK modulation. This value can be found for nonorthogonal CPFSK by numerically integrating the powerspectrum equations [1] and are valid for frequency-hopping signals provided that the number of symbols per dwell interval is large [2]. When a code of rate $R$ is used, the bandwidth becomes $B_{c}=B_{u} / R$. Since $B_{c} \leq B_{\max }$ is required, the minimum code rate that achieves the bandwidth constraint is $R_{\min }=B_{u} / B_{\max }$.

Guidance in the selection of the best values of $h, q$, and $R \geq$ $R_{\min }$ is provided by information theory. For specific values of $h$ and $q$, we evaluate the capacity $C(\gamma)$ as a function of $\gamma=\mathcal{E}_{s} / N_{o}$ under a bandwidth constraint for both the Rayleigh and AWGN channels. Since the noncoherent demodulator will include channel estimation, perfect channel-state information is assumed. Symbols are drawn from the signal set with equal probability. With these assumptions, a change of variables with $\mathbf{u}=\mathbf{y}_{i} / \sqrt{\mathcal{E}_{s}}$, and (17), the capacity for the fading channel may be expressed as [3]

$$
\begin{aligned}
C(\gamma) & =\log _{2} q-\frac{1}{q} \sum_{\nu=1}^{q} \iint p(a) p(\mathbf{u} \mid \nu, a) \\
& \times \log _{2} \frac{\sum_{k=1}^{q} I_{0}\left(2 a \gamma\left|u_{k}\right|\right)}{I_{0}\left(2 a \gamma\left|u_{\nu}\right|\right)} d \mathbf{u} d a
\end{aligned}
$$




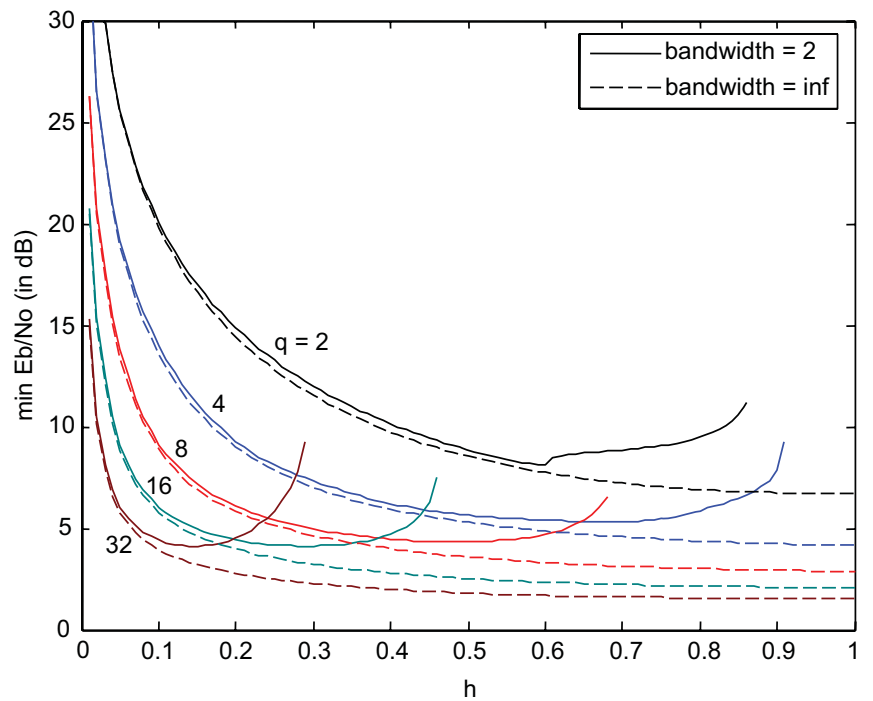

Fig. 2. Minimum $\mathcal{E}_{b} / N_{o}$ versus $h$ for the AWGN channel, $2 \leq q \leq 32$, $B_{\max } T_{b}=2$, and $B_{\max } T_{b}=\infty$.

where $p(a)$ is the density of the fading amplitude, the $(2 q+$ 1 )-fold integration is over all values of $a$ and the $2 q$ real and imaginary components of $\mathbf{u}$, and

$$
p(\mathbf{u} \mid \nu, a)=\frac{\gamma^{q} \exp \left[-\gamma\left(\mathbf{u}^{H} \mathbf{K}^{-1} \mathbf{u}+a^{2}\right)\right]}{\pi^{q} \operatorname{det} \mathbf{K}} I_{0}\left(2 a \gamma\left|u_{\nu}\right|\right) .
$$

Equation (30) is numerically integrated by the Monte Carlo method. To determine the minimum $\mathcal{E}_{b} / N_{o}$ necessary to maintain $C(\gamma)$ above the code rate $R$, we use the relationship $\mathcal{E}_{s}=R \mathcal{E}_{b} \log _{2} q$ and solve the equation

$$
R=C\left(R \mathcal{E}_{b} \log _{2} q / N_{o}\right)
$$

for all code rates such that $R_{\min } \leq R \leq 1$. For noncoherent systems under severe bandwidth constraints, the $R$ that minimizes $\mathcal{E}_{b} / N_{o}$ will typically be $R=R_{\min }$, but under loose bandwidth constraints the $R$ that minimizes $\mathcal{E}_{b} / N_{o}$ could possibly be larger than $R_{\min }$ (in which case the actual bandwidth is less than $B_{\max }$ ).

Figures 2 and 3 show plots of the minimum $\mathcal{E}_{b} / N_{o}$ versus $h$ for $2 \leq q \leq 32, B_{\max } T_{b}=2$, and $B_{\max } T_{b}=\infty$. Fig. 2 is for the AWGN channel, and Fig. 3 is for the Rayleigh fading channel. When $B_{\max } T_{b}=2$, the curves are truncated because there is a maximum value of $h$ beyond which no code exists that satisfies the bandwidth constraint. For each value of $q$, in each figure there is an optimal value of $h$ that gives the smallest value of the minimum $\mathcal{E}_{b} / N_{o}$. This smallest value decreases with $q$, but there are diminishing returns and the implementation complexity increases rapidly for $q>8$. Let $f_{e}$ denote the offset in the estimated carrier frequency at the receiver due to the Doppler shift and the frequency-synthesizer inaccuracy. The separation between adjacent frequencies in a CPFSK symbol is $h f_{b} / R \log _{2} q$, where $f_{b}$ denotes the information-bit rate. Since this separation must be much larger than $f_{e}$ if the latter is to be negligible as assumed in (4),

$$
f_{e}<<\frac{h f_{b}}{R \log _{2} q}
$$

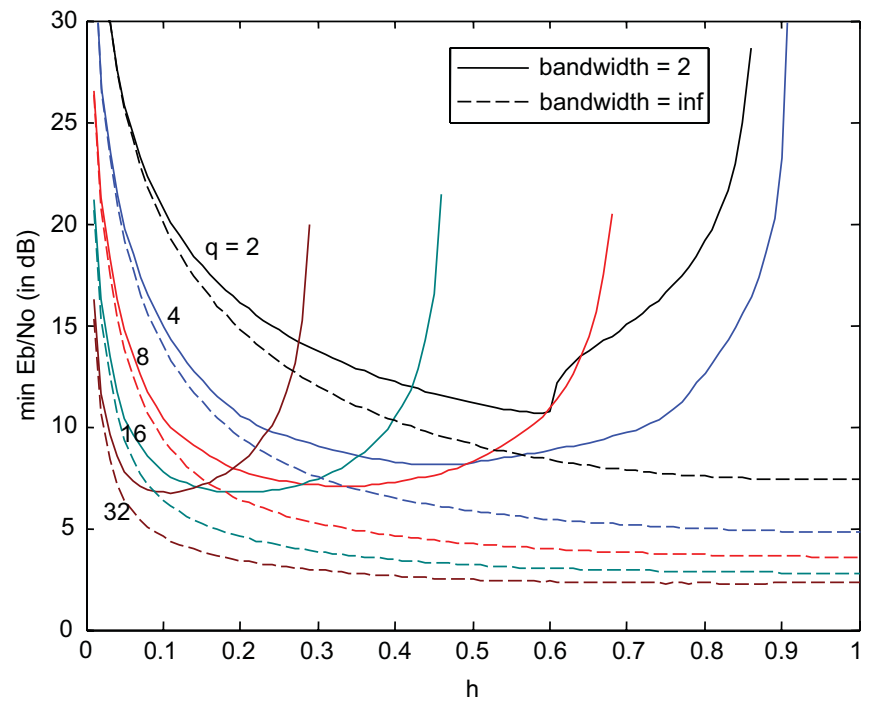

Fig. 3. Minimum $\mathcal{E}_{b} / N_{o}$ versus $h$ for the Rayleigh channel, $2 \leq q \leq 32$, $B_{\max } T_{b}=2$, and $B_{\max } T_{b}=\infty$.

is required. Since the optimal $h$ decreases while $R \log _{2} q$ increases with $q$, (33) is another reason to choose $q \leq 8$. For $q=4$ in Fig. 3, $h=0.46$ is the approximate optimal value when $B_{\max } T_{b}=2$, and the corresponding code rate is approximately $R=16 / 27$. For $q=8, h=0.32$ is the approximate optimal value when $B_{\max } T_{b}=2$, and the corresponding code rate is approximately $R=8 / 15$. For both $q=8$ and $q=4,(33)$ is satisfied if $f_{e}<<0.2 f_{b}$. At the optimal values of $h$, the plots indicate that the loss is less than $1 \mathrm{~dB}$ for the AWGN channel and less than $2 \mathrm{~dB}$ for the Rayleigh channel relative to what could be attained with the same value of $q, h=1$ (orthogonal CPFSK), and an unlimited bandwidth.

\section{Vi. Performance in Partial-Band Interference}

Simulation experiments were conducted to assess the benefits and tradeoffs of using the proposed nonorthogonal CPFSK coded modulation and accompanying channel estimator in a frequency-hopping system that suppresses partial-band interference. Interference is modeled as additional Gaussian noise within a fraction $\mu$ of the hopping band. The density of the interference (i.e., additional noise) is $I_{t 0} / \mu$, where $I_{t 0}$ is the spectral density when $\mu=1$ and the total interference power is conserved as $\mu$ varies. The parameter $A$ represents the spectral density due to the noise and the interference during a dwell interval. The bandwidth is assumed to be sufficiently small that the fading is flat within each frequency channel, and hence the symbols of a dwell interval undergo the same fading amplitude. The fading amplitudes are independent from hop to hop, which models the frequency-selective fading that varies after each hop. A block coincides with a dwell interval, and hence is suitable for the estimation of a single fading amplitude. Three alphabet sizes are considered: binary $(q=2)$, quaternary $(q=4)$, and octal $(q=8)$.

The simulated system uses the widely deployed turbo code from the UMTS specification [13], which has a constraint length of 4 , a specified code-rate matching algorithm, and 


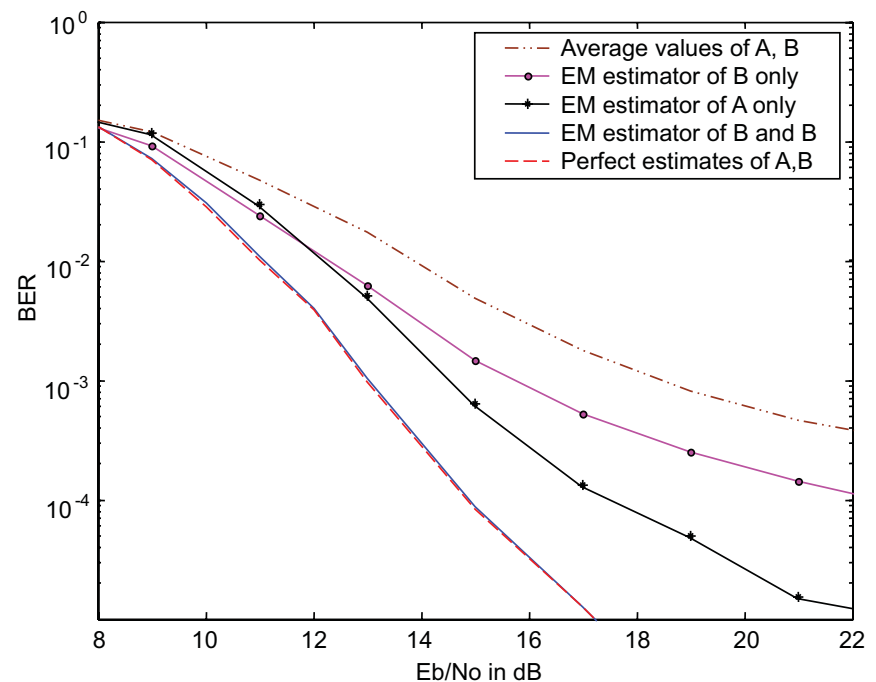

Fig. 4. Bit error rates of systems with various estimators in Rayleigh block fading with partial-band interference, $\mu=0.6, \mathcal{E}_{b} / I_{t 0}=13 \mathrm{~dB}$, turbo-coded octal CPFSK, $h=0.32$, code rate 2048/3840, and 32 hops per codeword.

an optimized variable-length interleaver that is set to 2048 . Simulation experiments with both turbo and convolutional codes of various constraint lengths indicate that the selected code provides close to the best performance in Rayleigh fading, although a constraint-length 3 is actually slightly better than the constraint-length 4 turbo code. However, in interference the performance difference is negligible. Since there is no standardized turbo code of constraint-length 3 with an optimized interleaver, the standardized UMTS code was selected for the simulated system in this section and the next one. If the dwell time is fixed but the codeword length is extended beyond the specified 2048 bits, then the number of hops per codeword will increase. As a result, the diversity order will increase, but the benefit of increased diversity order obeys a law of diminishing returns, and the benefit is minor at the bit error rates of interest (approximately $10^{-3}$ ). The values of modulation index $h$ and code rate $R$ are selected to be close to the information-theoretic optimal values given in Section $\mathrm{V}$ for Rayleigh fading under the bandwidth constraint $B_{\max } T_{b}=2$. In particular, a system with $q=2$ uses $h=0.6$ and $R=2048 / 3200$, one with $q=4$ uses $h=0.46$ and $R=2048 / 3456$, and one with $q=8$ uses $h=0.32$ and $R=2048 / 3840$. A receiver iteration comprises the steps of channel estimation, demapping, and one full turbo-decoding iteration. Up to 20 receiver iterations are executed. An early halting routine stops the iterations once the data is correctly decoded (which can be determined, for instance, by using the CRC specified in the UMTS standard). The number of hops per codeword may vary, and results below show the impact of changing this value.

Fig. 4 illustrates the influence of channel estimation on the bit error rate (BER) of the system. For this figure, $\mu=0.6$, $\mathcal{E}_{b} / I_{t 0}=13 \mathrm{~dB}$, the channel undergoes block-by-block Rayleigh fading, and octal CPFSK is used with 32 hops per codeword. The uppermost curve in the figure shows the performance of a simple system that does not attempt to estimate $A$ or $B$. Instead, the system sets these values to their statistical

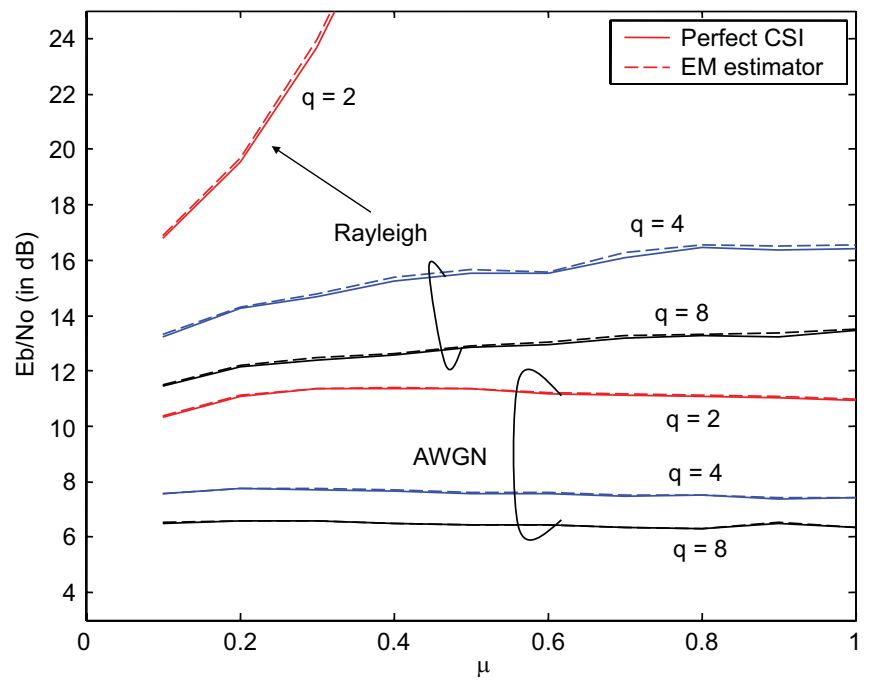

Fig. 5. Energy efficiency of binary $(q=2)$, quaternary $(q=4)$, and octal $(q=8)$ turbo-coded CPFSK in both AWGN and Rayleigh fading channels with a common bandwidth constraint $B_{\max } T_{b}=2$. For each combination of $q$ and channel type, the minimum $\mathcal{E}_{b} / N_{o}$ required to achieve BER $=10^{-3}$ as a function of $\mu$ is shown assuming both perfect CSI and the proposed EM estimator. There are 32 hops per codeword, and $\mathcal{E}_{b} / I_{t 0}=13 \mathrm{~dB}$. For binary CPFSK, $h=0.6$ and the code rate is $2048 / 3200$. For quaternary CPFSK, $h=0.46$ and the code rate is $2048 / 3456$. For octal CPFSK, $h=0.32$ and code rate is $2048 / 3840$.

averages: $A=N_{0}+I_{t 0}$, and $B=2 E[a] \sqrt{\mathcal{E}_{s}}=\sqrt{\pi \mathcal{E}_{s}}$. It can be seen that the performance of such a system is rather poor, as it has no knowledge of which hops have experienced interference and which have not. The system can be improved by estimating $A$ and/or $B$ on a block-by-block basis using the proposed EM estimator. The second curve from the top shows the performance when only $B$ is estimated on a blockby-block basis (and $A=N_{0}+I_{t 0}$ ), while the next curve down shows the performance when only $A$ is estimated on a blockby-block basis (and $B=\sqrt{\pi \mathcal{E}_{s}}$ ). The second lowest curve shows the performance when both $A$ and $B$ are estimated on a block-by-block basis with the proposed EM estimator, while the lowest curve shows the performance with perfect channel-state information (CSI), i.e., when $A$ and $B$ are known perfectly. As can be seen, there is a large gap between perfect CSI and simply using the average values of $A$ and $B$. This gap can be partially closed by estimating either $A$ and $B$ independently on a block-by-block basis, and the gap closes almost completely by estimating them jointly.

Fig. 5 illustrates the robustness of the estimator as a function of the alphabet size, channel type, and fraction of partial-band interference $\mu$. The figure shows the value of $\mathcal{E}_{b} / N_{0}$ required to achieve a BER of $10^{-3}$ as a function of $\mu$ for several systems with $\mathcal{E}_{b} / I_{t 0}=13 \mathrm{~dB}$. For each of the three alphabet sizes, both AWGN and block Rayleigh fading (again, 32 hops per codeword) are considered. For each of these six cases, the performance using perfect CSI and the performance with the proposed EM estimator are shown. Across the entire range of tested parameters, the proposed estimator's performance nearly matches that of perfect CSI. The benefit of increasing the alphabet size is apparent. For instance, in AWGN, increasing $q$ from 2 to 4 improves performance by about $4 \mathrm{~dB}$ while increasing it again from 4 to 8 yields another $1.2 \mathrm{~dB}$ gain. The 


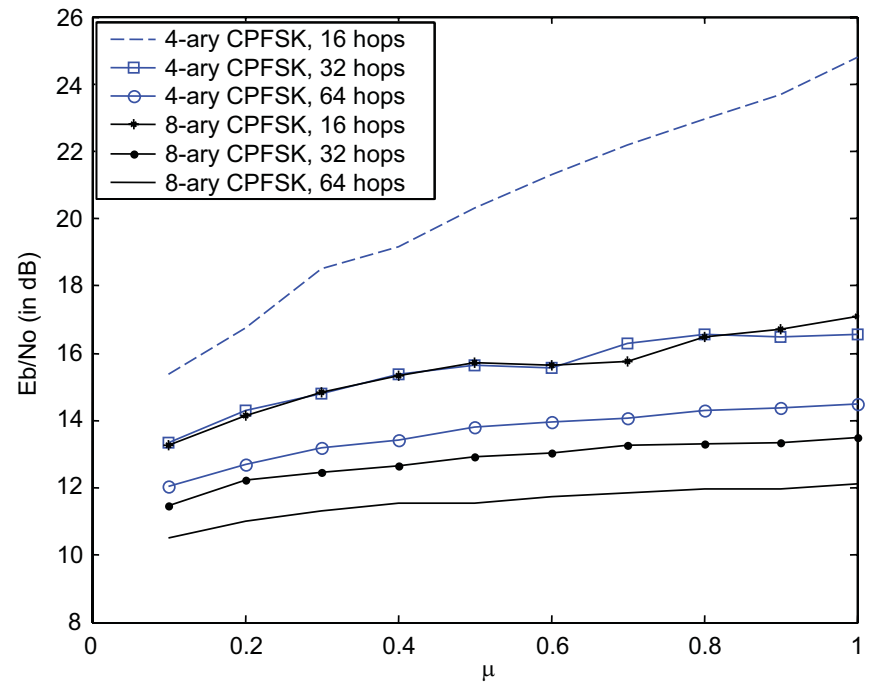

Fig. 6. Influence of the number of hops per codeword on the energy efficiency of quaternary and octal CPFSK in Rayleigh block fading with partial-band interference and $\mathcal{E}_{b} / I_{t 0}=13 \mathrm{~dB}$. Curves show the minimum $\mathcal{E}_{b} / N_{o}$ required to achieve $B E R=10^{-3}$ vs. $\mu$ using EM estimation of $A$ and $B$. The number of hops per codeword is 16,32 , or 64 . For $q=4$, the modulation index is $h=0.46$ and the code rate is $2048 / 3456$, resulting in 108,54 , or 27 symbols per hop. For $q=8, h=0.32$ and the code rate is $2048 / 3840$, resulting in 80,40 , or 20 symbols per hop.

gains in Rayleigh fading are even more dramatic. Although the performance in AWGN is relatively insensitive to the value of $\mu$, the performance in Rayleigh fading degrades as $\mu$ increases, and when $q=2$, this degradation is quite severe.

If the hop rate increases, the increase in the number of independently fading dwell intervals per codeword implies that more diversity is available in the processing of a codeword. However, the shortening of the dwell interval makes the channel estimation less reliable by providing the estimator with fewer samples. The influence of the number of hops per codeword is shown in Fig. 6 as a function of $\mu$ for quaternary and octal CPFSK using the EM estimator, Rayleigh block fading, and partial-band interference with $\mathcal{E}_{b} / I_{t 0}=13 \mathrm{~dB}$. Since the codeword length is fixed for each $q$, increasing the number of hops per codeword results in shorter blocks. For $q=4$, there are 108,54 , or 27 symbols per hop when there are 16,32 , or 64 hops per codeword, respectively. For $q=8$, there are 80,40 , or 20 symbols per hop when there are 16,32 , or 64 hops per codeword, respectively. Despite the slow decline in the accuracy of the EM channel estimates, the diversity improvement is sufficient to produce an improved performance as the number of code symbols per hop decreases. However, decreasing to fewer than 20 code symbols per hop will begin to broaden the spectrum significantly [2] unless the parameter values are changed.

Existing fielded frequency-hopping systems, such as GSM, Bluetooth, and combat net radios, use binary minimum-shift keying (MSK) with $\mathrm{h}=0.5$ or binary Gaussian FSK, and do not have fading-amplitude estimators. Figures 5 and 4 illustrate the substantial performance penalties resulting from the use of a binary modulation and the absence of fadingamplitude estimation, respectively. The cost of the superior performance of the proposed robust system is primarily the increased computational requirements, as discussed in Section IV. The frequency-hopping systems proposed in [5] and [6] are nonbinary and use channel estimators to achieve an excellent performance against partial-band interference and AWGN. However, they are not resistant to multiple-access interference because the transmitted symbols are not spectrally compact and the channel estimators are not designed to estimate multiple interference and noise spectral-density levels. As described in Section VII, the proposed robust system accommodates substantial multiple-access interference.

\section{AsynCHRONOUS Multiple-ACCESS InTERFERENCE}

Multiple-access interference may occur when two or more frequency-hopping signals share the same physical medium or network, but the hopping patterns are not coordinated. A collision occurs when two or more signals using the same frequency channel are received simultaneously. Since the probability of a collision in a network is decreased by increasing the number of frequency channels in the hopset, a spectrally compact modulation is highly desirable when the hopping band is fixed.

Simulation experiments were conducted to compare the effect of the number of users of a peer-to-peer network on systems with different values of $q$ and $h$. All network users have asynchronous, statistically independent, randomly generated hopping patterns. Let $T_{i}$ denote the random variable representing the relative transition time of frequency-hopping interference signal $i$ or the start of its new dwell interval relative to that of the desired signal. The ratio $T_{i} / T_{s}$ is uniformly distributed over the integers in $\left[0, N_{h}-1\right]$, where $N_{h}$ is the number of symbols per dwell interval, and it is assumed that the switching time between dwell intervals is negligible. Let $M$ denote the number of frequency channels in the hopset shared by all users. Since two carrier frequencies are randomly generated by each interference signal during the dwell interval of the desired signal, the probability is $1 / M$ that the interference signal collides with the desired signal before $T_{i}$, and the probability is $1 / M$ that the interference signal collides with the desired signal after $T_{i}$. Each interference signal transmits a particular symbol with probability $1 / q$ (common values of $q$ and $h$ are used throughout the network). The response of each matched filter to an interference symbol is given by the same equations used for the desired signal. The soft-decision-metrics sent to the decoder are generated in the usual manner but are degraded by the multiple-access interference.

The transmit power of the interference and the desired signals are the same. All the interference sources are randomly located at a distance from the receiver within 4 times the distance of the desired-signal source. All signals experience a path loss with an attenuation power law equal to 4 and independent Rayleigh fading. The interference signals also experience independent shadowing [2] with a shadow factor equal to $8 \mathrm{~dB}$.

The simulations consider CPFSK alphabet sizes from the set $q=\{2,4,8\}$. The hopping band has the normalized bandwidth $W T_{b}=2000$. Both orthogonal and nonorthogonal modulation are considered. For the orthogonal case, the code rate is chosen 


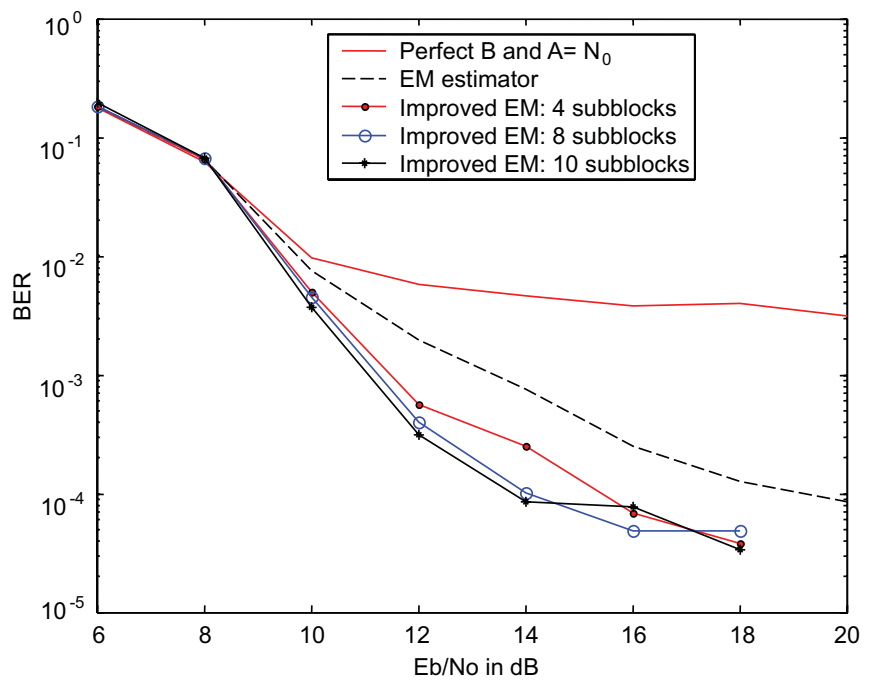

Fig. 7. Bit error rates of systems with various estimators in Rayleigh block fading with multiple-access interference created by 30 users. Turbo-coded octal CPFSK is used with $h=0.32$, code rate 2048/3840, and 32 hops per codeword. There are 1000 frequency channels.

to be $2048 / 6144$, which is close to the information-theoretic optimal value in Rayleigh fading when $h=1$. Taking into account the $99 \%$ power bandwidth of the resulting signal, there are $M=312,315$, and 244 frequency channels for binary, quaternary, and octal orthogonal CPFSK, respectively. For the nonorthogonal case, a bandwidth constraint $B_{\max } T_{b}=2$ is assumed so that there are $M=1000$ frequency channels for each $q$. As in the previous section, values of $h$ and $R$ that are close to the information-theoretic optimal values for this bandwidth constraint are selected (i.e. $h=0.6$ and $R=2048 / 3200$ for $q=2, h=0.46$ and $R=2048 / 3456$ for $q=4$, and $h=0.32$ and $R=2048 / 3840$ for $q=8$ ). In all cases, there are 32 hops per codeword.

In the presence of multiple-access interference, it is important to accurately estimate the values of $A$ and $B$. The impact of the channel estimation technique is illustrated in Fig. 7 for a system with 30 users all transmitting nonorthogonal octal CPFSK. The uppermost curve shows what happens when the receiver ignores the presence of interference. In this case, $B$ is set to its actual value (perfect CSI for $B$ ), while $A$ is set to $N_{0}$, its value without interference. Performance can be improved by using the proposed EM-based estimator for jointly estimating $A$ and $B$ on a block-by-block basis, as illustrated by the second curve from the top.

Unlike the partial-band interference case, where the value of $A$ is constant for the entire duration of the hop, the value of $A$ in the presence of multiple-access interference will not generally be constant due to the asynchronous hopping. This fact suggests that performance can be improved by partitioning the block into multiple sub-blocks, and obtaining a separate estimate of $A$ for each sub-block. Such estimates can be found from a simple modification of the proposed EM-based estimator. The estimator first finds the value of $B$ for the entire block from (26) as before. Next, it finds the value of $A$ for each sub-block from (25) using the value of $B$ found for the entire block and the value of $D$ for just that sub-block (with

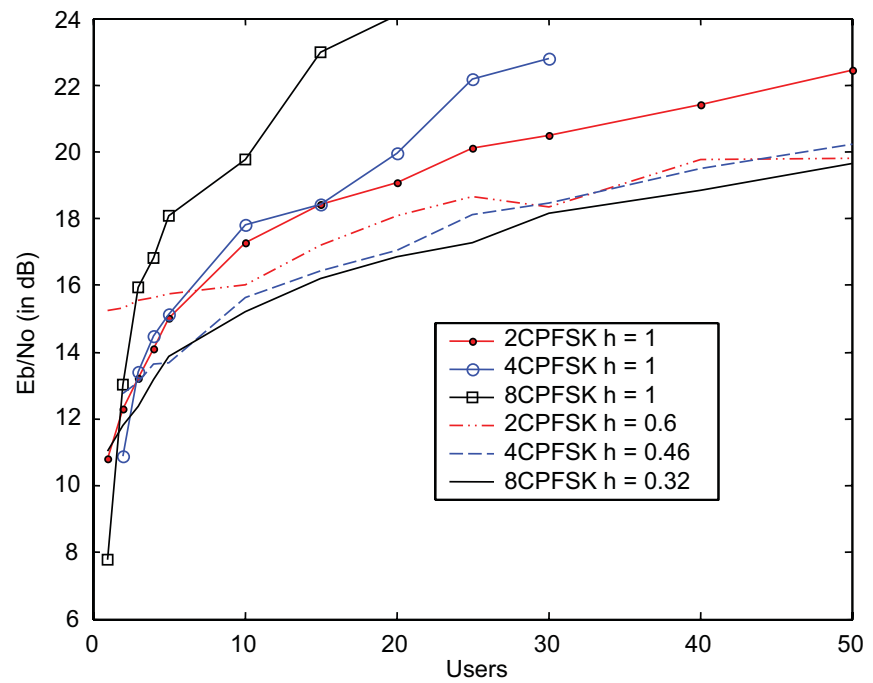

Fig. 8. Energy efficiency of binary, quaternary, and octal turbo-coded CPFSK in the presence of multiple-access interference and Rayleigh block fading (32 hops per codeword). The curves show the minimum $\mathcal{E}_{b} / N_{o}$ required to achieve $B E R=10^{-4}$ as a function of the number of users. For each $q$, curves are shown for both orthogonal and nonorthogonal signaling. A hopping bandwidth $W T_{b}=2000$ is assumed for each case. When nonorthogonal signaling is used, a bandwidth constraint $B_{\max } T_{b}=2$ is imposed so that there are 1000 frequency channels (the code rates and modulation indices are the same as in Fig. 6). When orthogonal signaling is used, the code rate is 2048/6144, and there are 312, 315, and 244 frequency channels for binary, 4-ary, and 8-ary FSK, respectively.

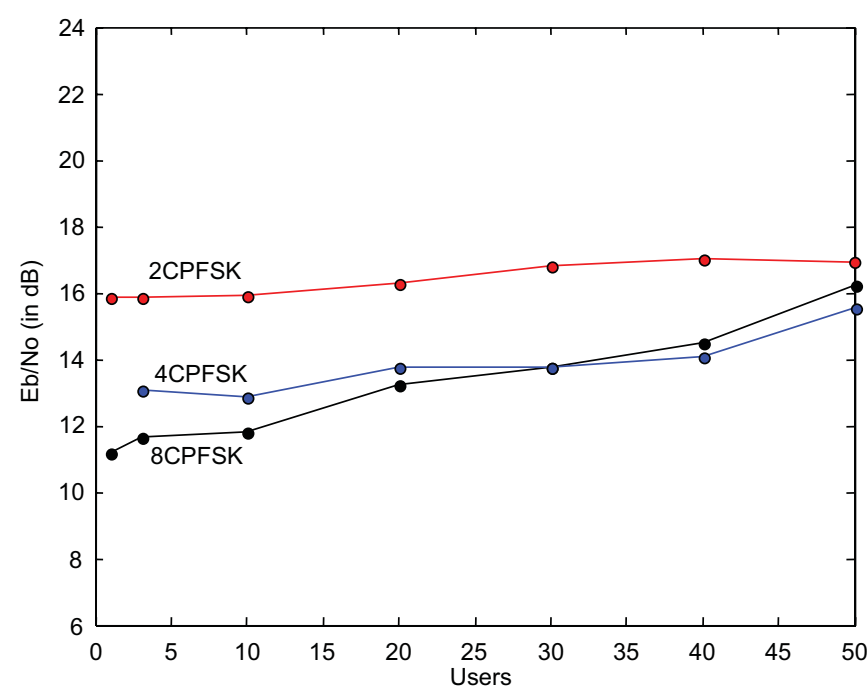

Fig. 9. Energy efficiency using improved EM estimation of nonorthogonal binary, quaternary, and octal turbo-coded CPFSK in the presence of multipleaccess interference and Rayleigh block fading. The curves show the minimum $\mathcal{E}_{b} / N_{o}$ required to achieve $B E R=10^{-4}$ as a function of the number of users. The system parameters are the same as for the nonorthogonal cases in Fig. 8. For $q=2$ and $q=8$, there are 10 sub-blocks, and for $q=4$ there are 9 .

$N$ set to the size of the sub-block). The bottom three curves in Fig. 7 show the performance when 4, 8, or 10 sub-blocks are used to estimate $A$. While it is beneficial to use more subblocks at low $\mathcal{E}_{b} / N_{o}$, at higher $\mathcal{E}_{b} / N_{o}$ even using only 4 subblocks is sufficient to give significantly improved performance. This method of sub-block estimation entails essentially no additional complexity. 
Figures 8 and 9 show the energy efficiency as a function of the number of users. In Fig. 8, the performance using the block EM estimator (no sub-block estimation) is shown. In particular, the minimum required value of $\mathcal{E}_{b} / N_{o}$ to achieve a bit error rate equal to $10^{-4}$ is given as a function of the number of users. The performance is shown for both orthogonal and nonorthogonal modulation and the three values of $q$. For a lightly loaded system (less than five users), the orthogonal systems outperform the nonorthogonal ones because orthogonal modulation is more energy efficient in the absence of interference. However, as the number of users increases beyond about five, the nonorthogonal systems offer superior performance. The reason is that the improved spectral efficiency of a nonorthogonal modulation allows more frequency channels, thereby decreasing the probability of a collision. With orthogonal modulation, performance as a function of the number of users degrades more rapidly as $q$ increases because larger values of $q$ require larger bandwidths. In contrast, with nonorthogonal modulation, the best performance is achieved with the largest value of $q$, although performance with $q=2$ or $q=4$ is only about $1-2 \mathrm{~dB}$ worse than with $q=8$. When there are 50 users, nonorthogonal CPFSK with $q=8$ is about $3 \mathrm{~dB}$ more energy efficient than the more conventional orthogonal CPFSK with $q=2$.

Fig. 9 shows the performance with nonorthogonal CPFSK when sub-block estimation is used instead of block estimation. For $q=8$ there are 10 sub-blocks of $40 / 10=4$ symbols, for $q=4$ there are 9 sub-blocks of $54 / 9=6$ symbols, and for $q=2$ there are 10 sub-blocks of $100 / 10=10$ symbols. A comparison with Fig. 8 indicates that for $q=8$ and 50 users, there is a $4 \mathrm{~dB}$ gain in energy efficiency relative to the block estimator. It is also observed that when using the sub-block estimator, the performance is less sensitive to the number of users, and that in a very lightly loaded system, the block estimator offers better performance (since then $A$ is likely to be constant for the entire hop).

\section{CONCLUSIONS}

A noncoherent frequency-hopping system with nonorthogonal CPFSK has been designed to be highly robust in environments including frequency-selective fading, partial-band interference, multitone jamming, and multiple-access interference. The robustness is due to the iterative turbo decoding and demodulation, the channel estimator based on the expectationmaximization algorithm, and the spectrally compact modulation.

\section{REFERENCES}

[1] J. G. Proakis, Digital Communications, 4th ed. New York: McGraw-Hill, 2001.

[2] D. Torrieri, Principles of Spread-Spectrum Communication Systems. Boston: Springer, 2005.

[3] G. Caire, G. Taricco, and E. Biglieri, "Bit-interleaved coded modulation," IEEE Trans. Inform. Theory, vol. 44, pp. 927-946, May 1998.
[4] Q. Zhang and T. Le-Ngoc, "Turbo product codes for FH-SS with partialband interference," IEEE Trans. Wireless Commun., vol. 1, pp. 513-520, July 2002.

[5] W. G. Phoel, "Iterative demodulation and decoding of frequency-hopped PSK in partial-band jamming," IEEE J. Select. Areas Commun., vol. 23, pp. 1026-1033, May 2005.

[6] H. El Gamal and E. Geraniotis, "Iterative channel decoding and estimation for convolutionally coded anti-jam FH signals," IEEE Trans. Commun., vol. 50, pp. 321-331, Feb. 2002.

[7] K. R. Narayanan and G. L. Stuber, "Performance of trellis-coded CPM with iterative demodulation and decoding," IEEE Trans. Commun., vol. 49, pp. 676-687, Apr. 2001.

[8] M. C. Valenti and S. Cheng, "Iterative demodulation and decoding of turbo coded M-ary noncoherent orthogonal modulation," IEEE J. Select. Areas Commun., vol. 23, pp. 1739-1747, Sept. 2005.

[9] S. Benedetto, D. Divsalar, G. Montorsi, and F. Pollara, "A soft-input soft-output APP module for iterative decoding of concatenated codes," IEEE Commun. Lett., vol. 1, pp. 22-24, Jan. 1997.

[10] G. J. McLachlan and T. Krishnan, The EM Algorithm and Extentions. Wiley, 1997.

[11] S. Cheng, M. C. Valenti, and D. Torrieri, "Robust iterative noncoherent reception of coded FSK over block fading channels," IEEE Trans. Wireless Commun., vol. 6, pp.3142-3147, Sept. 2007.

[12] S. C. Chapra and R. Canale, Numerical Methods for Engineers, 4th ed. New York: McGraw-Hill, 2002.

[13] Third Generation Partnership Project (3GPP), "Universal mobile telecommunications system (UMTS): Multiplexing and channel coding (FDD)," 3GPP TS 25.212 Version 6.6.0 Release 6, Sept. 2005.

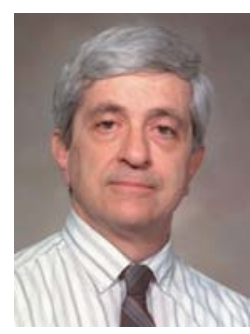

Don Torrieri is a research engineer and Fellow of the US Army Research Laboratory. His primary research interests are communication systems, adaptive arrays, and signal processing. He received the $\mathrm{Ph}$. D. degree from the University of Maryland. He is the author of many articles and several books including Principles of Spread-Spectrum Communication Systems (Springer, 2005). He teaches graduate courses at Johns Hopkins University and has taught many short courses. In 2004, he received the Military Communications Conference achievement award for sustained contributions to the field.

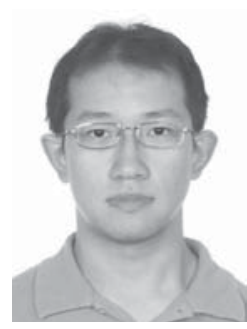

Shi Cheng received the B.E. and M.S. degrees in electrical engineering from Southeast University, Nanjing, China in 2000 and 2003 respectively, and the $\mathrm{Ph} . \mathrm{D}$. degree in electrical engineering from West Virginia Unversity, Morgantown, WV in 2007. He is currently a system research engineer in ArrayComm LLC, San Jose, CA. His research interests lie in the areas of information theory, coding theory, and communications signal processing.

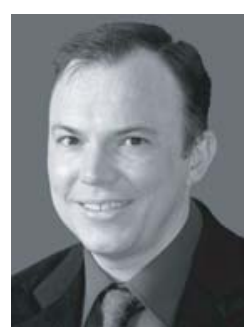

Matthew C. Valenti holds BS and Ph.D. degrees in Electrical Engineering from Virginia Tech. From 1992 to 1995 he was an electronics engineer at the US Naval Research Laboratory, at which time he earned a MS in electrical engineering from the Johns Hopkins University. Since 1999, he has been with West Virginia University, where he is currently an Associate Professor in the Lane Department of Computer Science and Electrical Engineering. He serves as an associate editor for IEEE TRANSACTIONS ON WIRELESS COMMUNICATIONS, and has served as a track co-chair for the Fall 2007 Vehicular Technology Conference (Baltimore, Maryland) and the 2009 International Conference on Communications (Dresden, Germany). His research interests are in the areas of communication theory, error correction coding, applied information theory, and wireless networks. 